\title{
Siglec-1 Expressed on Dendritic Cells is a New Receptor Implicated in Arenavirus Uptake ${ }^{\dagger}$
}

\author{
Xabier Muniz-Trabudua 1,2, Cristina Borio ${ }^{3}$, Marcos Bilen ${ }^{3}$, Itziar Erkizia ${ }^{1}$, Daniel Perez-Zsolt ${ }^{1,2}$, \\ Susana Benet 1,2, Javier Martinez-Picado 1,4,5,* and Nuria Izquierdo-Useros 1,6,* \\ 1 IrsiCaixa AIDS Research Institute, 08916 Badalona, Spain; xmuniz@irsicaixa.es (X.M.-T.); \\ Ierkizia@irsicaixa.es (I.E.); dperez@irsicaixa.es (D.P.-Z.); sbenet@irsicaixa.es (S.B.) \\ 2 Departamento de Biología Celular, Fisiología e Inmunología, Universitat Autònoma de Barcelona, \\ 08913 Barcelona, Spain \\ 3 Laboratorio de Ingeniería Genética y Biología Celular y Molecular-Area Virosis Emergentes y Zoonóticas, \\ Department of Science and Technology, UniversidadNacional de Quilmes, Bernal, \\ B1876 Buenos Aires, Argentina; cborio@unq.edu.ar (C.B.); mbilen@unq.edu.ar (M.B.) \\ 4 Chair in Infectious Diseases and Immunity, Centre for Health and Social Care Research (CESS), Faculty of \\ Medicine, University of Vic-Central University of Catalonia (UVic-UCC), 08500 Vic, Spain \\ 5 Catalan Institution for Research and Advanced Studies (ICREA), 08010 Barcelona, Spain \\ 6 Institut d'Investigació en Ciències de la Salut Germans Trias i Pujol, 08916 Badalona, Spain \\ * Correspondence: Jmpicado@irsicaixa.es (J.M.-P.); nizquierdo@irsicaixa.es (N.I.-U.) \\ † Presented at Viruses 2020-Novel Concepts in Virology, Barcelona, Spain, 5-7 February 2020. \\ Published: 19 June 2020
}

\begin{abstract}
Arenaviruses are enveloped viruses that cause hemorrhagic fever outbreaks in humans and still lack an effective antiviral treatment. Upon early infection, these viruses target dendritic cells (DCs), which can promote systemic viral dissemination, contributing to pathogenesis. We have previously described that Siglec-1, a sialic acid Ig-like binding lectin-1 expressed on DCs interacts with different enveloped viruses and promotes their capture within a virus-containing compartment. Such is the case of HIV-1 or Ebola virus, which display sialylated gangliosides on their viral envelope that are effectively recognized by Siglec- 1 . Here, we aimed to study if Siglec-1 on DCs also interacts with arenaviruses such as Junin. We produced non-infectious Junin viral-like particles (Junin-VLPs) tagged with fluorescent Egfp by transfecting a plasmid encoding the structural Junin Z protein on HEK-293T cells. Junin-VLPs were added to a Raji cell line stably transfected with Siglec-1 or to monocyte-derived DCs activated or not with either Interferon- $\alpha$ or lipopolysaccharide. Viral uptake was analyzed by FACS or confocal microscopy in the presence of an anti-Siglec-1 monoclonal antibody $(\mathrm{mAb})$ or an isotype control. Statistical differences were assessed with the indicated tests. Raji Siglec- 1 cells captured a higher number of Junin-VLPs than Raji cells, and this was blocked with an anti-Siglec-1 mAb ( $P=0.0159$; Mann-Whitney). On primary DCs, activation enhanced Junin-VLP capture $(P=0.0024$; paired $t$-test $)$ and Siglec- 1 expression. Furthermore, pre-incubation with an anti-Siglec-1 mAb on activated DCs blocked Junin-VLP uptake $(P \leq 0.0002$; one sample $t$-test), while an isotype control did not. Forty-nine percent of the activated DCs analyzed by confocal microscopy captured Junin-VLPs within a Siglec-1+ virus-containing compartment. Moreover, when HIV-1 was also added, $97 \%$ of those compartments retained both viruses. Thus, we conclude that Siglec-1 is a new receptor involved in arenavirus uptake in DCs and could represent a novel target for an anti-arenavirus treatment.
\end{abstract}

Keywords: arenavirus; dendritic cells; Siglec-1 
(C) 2020 by the authors. Licensee MDPI, Basel, Switzerland. This article is an open access article distributed under the terms and conditions of the Creative Commons Attribution (CC BY) license (http://creativecommons.org/licenses/by/4.0/). 